\title{
Tsafon
}

Revue d'études juives du Nord

$78 \mid 2019$

L'antijudaïsme dans l'Antiquité

\section{« Signe du temps » ou de l'esthétique du simple et du fort chez Rachel}

Bernard Grasset

\section{(2) OpenEdition}

1 Journals

Édition électronique

URL : https://journals.openedition.org/tsafon/2463

DOI : $10.4000 /$ tsafon. 2463

ISSN : 2609-6420

Éditeur

Association Jean-Marie Delmaire

Édition imprimée

Date de publication : 1 décembre 2019

Pagination : 109-124

ISSN : 1149-6630

\section{Référence électronique}

Bernard Grasset, « « Signe du temps » ou de l'esthétique du simple et du fort chez Rachel », Tsafon [En ligne], 78| 2019, mis en ligne le 01 décembre 2019, consulté le 27 juin 2021. URL : http:// journals.openedition.org/tsafon/2463; DOI : https://doi.org/10.4000/tsafon.2463

Tsafon. Revues d'études juives du Nord 


\section{Varia : Littérature}

\section{«Signe du temps » ou de l'esthétique du simple et du fort chez Rachel}

Bernard Grasset*

Dans un article, jamais traduit en français, la poétesse Rachel exprime avec ardeur et clarté sa conception de la poésie. Nous en proposons la traduction après avoir rappelé les étapes de sa courte vie et présenté l'art littéraire selon Rachel ${ }^{1}$.

\section{Rachel, sa vie, son œuvre}

\section{Une vie brève}

Rachel Blaustein, connue sous le prénom de Rachel choisi comme nom d'auteur en écho de la femme de Jacob, naît au début de l'automne 1890 à Saratov en Russie. Elle est la fille tardive d'Asir Blaustein, commerçant juste et résolu, et de Sophie Mandelstam, femme aimante, fidèle, dont le père était rabbin. Le milieu familial est imprégné de religion, mais aussi d'art, de littérature. Le frère aîné de Rachel, Jacob, est philosophe, l'une de ses sœurs, Bethsabée, musicienne. Quant à Rachel, elle rêve de devenir peintre. Alors que Rachel est encore enfant, la famille s'installe à Poltava, plus au sud. La disparition de Sophie, sa

\footnotetext{
* Membre associé de l'Équipe de recherche «La Bible et ses lectures », Université catholique de l'Ouest (Angers) - UMR 8167.

${ }^{1}$ Cet article «Sur le signe du temps », ainsi que d'autres articles littéraires de Rachel, ses poèmes épars et des extraits de sa correspondance, sont à paraitre à l'automne 2020 aux éditions Arfuyen qui ont déjà publié en 2006 Regain et en 2013 De loin suivi de Nébo dans notre traduction.
} 
mère, cause une vive blessure dans le cœur de Rachel. Passionnée de peinture, elle n'en écrit pas moins, à quinze ans, des poèmes en russe. En 1907, à dix-sept ans, avec sa famille elle connaît un nouvel exil, cette fois-ci à Kiev où elle fréquente pendant deux ans l'Académie des BeauxArts.

L'année 1909, alors que Rachel n'a pas encore vingt ans, elle part en voyage en Palestine. Fascinée par le pays ancestral, elle apprend la langue antique, l'hébreu, et renonce à parler en russe comme à revenir en Russie. Elle séjourne à Rehovot avant de rejoindre Kinnéret. Deux rencontres alors influencent le destin de Rachel : celle avec Anne Meizel qui l'initie au travail agricole, celle avec Aharon David Gordon qui devient comme son guide spirituel ${ }^{2}$. Après avoir espéré être admise à 1'Académie des Beaux Arts Betsalel de Jérusalem, elle organise avec sa sœur Suzanne des veillées musicales auxquelles participe Bethsabée.

En 1913, Rachel gagne la France, Toulouse, afin d'y entreprendre des études universitaires d'agronomie. Elle y travaille avec sérieux durant trois années, a pour seul loisir les promenades, rencontre Michaël Braunstein qui occupera une place importante dans sa vie sentimentale et éprouve une poignante nostalgie pour Tibériade.

La guerre la contraint de regagner la Russie en 1916 où elle travaille au service d'enfants de réfugiés. De premiers symptômes de maladie apparaissent. Si le rêve de devenir peintre s'éloigne, Rachel, qui vit à Odessa auprès de sa famille appauvrie, traduit en russe des poèmes de Bialik et écrit ses souvenirs sur la vie à Tibériade.

La guerre finie, en 1919, Rachel embarque sur le Rouslan et rejoint Kinnéret. Mais elle n'est plus la même, la jeunesse s'en est comme allée. Portant un vêtement sombre, propre, qui paraissait unique et ressemblait à celui d'une collégienne ou d'une religieuse, elle était comme courbée par la maladie. Ceux qui la rencontraient pressentaient la gravité de celleci. Son regard azuré, intense, interrogateur rappelait le calme de la mer avant le déchaînement de la tempête.

Rachel n'en travaille pas moins avec ardeur la terre au kibboutz Degania, près du lac, alors qu'on lui avait proposé un travail de professeur. Mais elle s'affaiblit peu à peu et sa tuberculose met en danger les autres membres du kibboutz. On lui annonce à regret qu'elle doit partir. Rachel parlera souvent de cette nuit : «Un nuage épais et noir est descendu sur moi, je voulais crier, mais je ne pouvais pas ». Rachel lutte,

\footnotetext{
${ }^{2}$ Dans l'une de ses lettres, ayant perçu en elle « un talent original », il l'encourage à être elle-même et à trouver le chemin qui mène, par le travail, à la lumière.
} 
enseigne l'agronomie, l'hébreu. Étrangère à la vie urbaine, elle n'en est pas moins contrainte en 1925 de vivre à Tel-Aviv où elle loue une petite chambre face à la mer, rue Bograshov, après avoir séjourné à Jérusalem et Safed. La vocation de peintre s'est évanouie comme celle de paysanne. Lui reste la vocation brûlante de poète ${ }^{3}$.

Rachel vit désormais dans la pauvreté, reçoit des amis, souffre et écrit. Elle doit rejoindre le sanatorium Bilou à Guedéra. "Chaque jour m'est comme une sorte de mort » écrit-elle dans une de ses notes ultimes. Elle veut retrouver sa chambre, face à la mer. Hospitalisée à Tel-Aviv, elle s'y éteint au printemps 1931. Elle n'avait pas encore quarante-et-un ans. Au cimetière de Tibériade, près du lac, sous le ciel azuré, elle repose. Ses amis plantent sur la colline des palmiers de Bagdad qui forment le Jardin de Rachel.

\section{Une ouvre féconde}

«La langue est souple, savoureuse, harmonieuse - notre langue hébraïque » écrivait Rachel en conclusion de son article Murmure des jours. Cette langue de la mémoire, elle l'avait apprise avec passion au contact des enfants, par les cours du professeur Yéhudah Teller, en lisant la Bible, lors de son arrivée en Palestine, tout en découvrant la beauté des paysages, de la lumière, du pays ancestral. Âgée d'un peu de plus de vingt ans, elle est ainsi passée du russe et du yiddish qu'elle connaissait à l'hébreu, avec ardeur, jusqu'à en acquérir une parfaite maîtrise et participer à la résurrection de cette langue, devenue seulement liturgique, dans la poésie. Apprendre l'hébreu, c'était une nouvelle naissance.

Enracinée dans la Bible, l'œuvre de Rachel a été influencée sur le plan littéraire par la poésie russe (Korolenko, Blok, Akhmatova), le mouvement acméiste (voir infra) et la poésie française (notamment Francis Jammes). L'une des marques distinctives de son style, qui se manifeste aussi bien dans ses poèmes que dans ses articles, est l'attachement à la simplicité. Alliée à la ferveur, la simplicité, comme d'enfance, atteint le cœur. L'écriture fervente devient écriture bouleversante. L'expression simple qui rejoint le cœur par son humaine vérité, qui s'élève à un sobre lyrisme, reste dans la mémoire gravée.

Il y a chez Rachel une essentielle humilité du langage. L'idéal de la poésie à ses yeux : être humaine, la plus humaine possible. Un souffle de pur lyrisme habite l'art de Rachel. Sa poésie, marquée par son

\footnotetext{
${ }^{3}$ Deux recueils ont paru de son vivant: Regain (1927), De loin (1930), le troisième, Nébo (1932), après sa mort.
} 
authenticité, enracinée dans la terre, l'origine, aspire à être accueillie par le peuple et non par les seules élites. Pour Rachel, le poète, l'artiste, l'écrivain sont appelés à traduire, avec lyrisme, émotion, notre condition humaine. Dans son article Expression lyrique, elle perçoit cette condition comme double : l'homme est à la fois un être prisonnier dans les chaînes de l'incompréhension, de la souffrance, de la mort, et un être de liberté, qui ne cesse de tendre à vivre pleinement libre, en harmonie, en paix avec autrui. Dans la poésie de l'auteur de Regain revient, comme un refrain, la conception de l'existence comme combat entre les forces d'asservissement et les forces de liberté. Celle qui était en quête de liberté disait: "Chaque homme a son Nébo ». Nous apercevons la plénitude sans pouvoir l'atteindre au sein de notre finitude. Écrire est recherche d'humanité et de lumière.

Rachel, qui rêve les hommes vrais, naturels, solidaires, se tourne vers la Bible pour y trouver la lumière sur l'existence, sa vie. Dès 1919, dans son premier article, poème en prose, Sur les rives de Tibériade, elle évoque "notre père Abraham », la parole psalmique de louange et, de manière inattendue, « la noble face du prédicateur de Nazareth ». Habiter le Pays c'est retrouver la mémoire. Son esthétique prise l'enracinement scripturaire de la symbolique poétique. La vertu stylistique qu'elle loue dans Sur le signe du temps prend sa source lointaine dans la Bible. Six poèmes de Rachel portent en exergue une citation biblique ${ }^{4}$. Quatre poèmes ont pour titre le nom d'un personnage biblique : Rachel, Mickal, Élie, Jonathan ${ }^{5}$. Des personnages de la Bible surgissent par ailleurs au sein des poèmes: Rachel, Anne, Salomon, Saül (non nommé mais évoqué), la fille de Jephté. Les lieux bibliques parcourent l'écriture poétique $^{6}$. Des versets scripturaires se mêlent aux vers (comme Lv 19, 18.34 sur l'amour du prochain, de l'étranger, dans le poème $\mathrm{Ne}$ me condamne pas). Le dix-neuvième poème de Nébo s'ouvre par le vers « Ma Bible est ouverte sur le livre de Job »?.

Rachel lisait la Bible, s'en nourrissait, rapprochait son expérience de celle des personnages bibliques. Le titre de son recueil De loin prend

\footnotetext{
${ }^{4}$ Deux poèmes citent les livres de Samuel, un le second livre des Rois, deux le Cantique des cantiques et un le livre de Job.

${ }^{5}$ Le titre du trente-sixième poème de Regain, Jour de bonne nouvelle, reprend la fin de la citation biblique en exergue. Le quatrième poème de Nébo, Shabbat, renvoie à l'univers biblique. Dans les Poèmes épars, le titre La brebis du pauvre est aussi biblique : $2 \mathrm{Sm} \mathrm{12,3-4.}$

${ }^{6}$ Nébo, Siloh, Samarie, Hermon, Guilboa, Golan, Jourdain, Mont des Oliviers et bien sûr Tibériade.

${ }^{7}$ Le mot Bible (Tanakh) apparaît également dans le poème Juive du même recueil.
} 
sa source dans le livre du Deutéronome ${ }^{8}$. À son dernier recueil, ses amis, dans le prolongement de De loin, ont donné le titre biblique de Nébo. Rachel s'identifie, dans sa condition de poète, à Moïse qui regarde, depuis le mont Nébo, la Terre promise sans pouvoir l'atteindre ${ }^{9}$. Le nom même d'auteur qu'a choisi Rachel est biblique : en portant ce nom, elle laisse la voix de l'épouse de Jacob chanter en elle.

Discrètement mais intensément, Dieu est présent dans l'œuvre de Rachel. C'est un Dieu créateur, d'alliance, familier au cœur, puissant, qui peut exiger beaucoup, s'irriter, frapper, mais aussi apaiser, prendre soin, sauver, dont la main se pose parfois sur les blessures et ouvre accès à la fête du printemps. Un Dieu contre lequel la poétesse se révolte, qu'elle ne comprend pas, un Dieu qu'elle accueille, loue, bénit. L'écriture, la vie de Rachel demeurent en quête de lointain. Comme le poète Shin Shalom sur lequel elle publie un article un an avant sa mort, elle a le sens du mystère et perçoit l'existence, en sa profondeur, comme un dialogue entre le cœur humain et le cœur divin. Nourrie de légendes, dans l'attente d'un salut, Rachel élève son regard jusqu'à l'horizon, l'infini. «[...] tu jettes un regard d'amour vers le lac ! Que de beauté ! Azur, azur, azur indicible, porteur de paix, guérison de l'âme [...]» écrit-elle avec lyrisme dans Sur les rives de Tibériade où elle souligne l'importance de la soif ${ }^{10}$ et du chant. Tout dans le poète doit chanter, tout doit être chanté par le poète, tout résonnera de son chant. Qui a des racines profondes et demeure assoiffé peut gravir les montagnes pour y respirer l'air des cimes, guetter l'aurore qui vient. Rachel, amie d'A. D. Gordon, aspire à une forme de relation absolue, à une extase mystique. Celle que l'on appelait «oiseau chanteur de Kinnéret» a chanté l'existence comme lutte, incessant combat, a chanté jusqu'à la mort dans l'espérance de la victoire de la vie.

\section{L'art littéraire : Sur le signe du temps}

Outre des recueils de poésie, Rachel est également l'auteur de vingt-et-un articles, essentiellement littéraires, écrits en hébreu entre

\footnotetext{
${ }^{8}$ Verset 32, 52, dont le début est cité sous le titre. Le dernier poème de ce recueil est intitulé De loin. Le mot Regain, titre du premier recueil, tire son origine de Lv 25, 5.

${ }^{9}$ Sur sa tombe sont gravés les quatre derniers vers du poème et du recueil De loin qui s'inspirent de Dt 32, 49-52 : «Tendre les mains. Voir de loin. / Là-bas - nul ne vient. / Un homme... Nébo / Devant une terre d'abondance ».

${ }^{10}$ « [...] quiconque en [1'eau de Tibériade] boira au moins une fois reviendra toujours vers elle », Shirat Rahel (Poésie de Rachel), Jérusalem, éditions 'Arièl, 2001, p. 200.
} 
1920 et 1930, hormis le premier, Sur les rives de Tibériade, écrit en russe en 1919. Au fur et à mesure de leur écriture, ils ont été publiés dans différents journaux et revues littéraires ${ }^{11}$. Ces articles, réunis pour la première fois dans l'édition de 1939 de la poésie de Rachel, occupent une trentaine de pages, comme ses traductions.

L'article littéraire Sur le signe du temps, publié dans le supplément de Davar au mois de nissan (mars/avril) 1927, apparaît comme l'un des plus importants qu'ait écrit Rachel, en tout cas l'un de ceux où s'exprime le mieux, avec le plus grand relief, son esthétique poétique, les principes qui la sous-tendent. L'auteur de Regain commence par définir ce qu'est, à ses yeux, le signe du temps ('ot hazeman). «[...] le signe du temps dans l'art poétique est la simplicité de l'expression (pashtout habitouy) ». La « simplicité d'expression » va devenir le fil directeur de l'article. Loin des «artifices littéraires », cette simplicité d'expression, directe, faite de dépouillement, se conjugue à un lyrisme de l'émotion. Elle « touche le cœur, nous dit Rachel, par sa vérité humaine ». N'est-ce pas cette capacité qui caractérise par excellence sa propre poésie ? Une poésie qui nous émeut par sa profondeur humaine. La force de la simplicité se grave dans la mémoire. Les mots simples, sobres, justes, qui accompagnent le temps des hommes, peuvent s'élever au chant de joie. Il en est ainsi de la poésie de Rachel.

Après avoir présenté les poètes de langues russe, française et hébraïque auxquels se réfère l'auteur de Regain, nous essaierons de faire ressortir les traits dominants de l'esthétique de Rachel tels qu'ils se dégagent de cet article et les principes de son jugement sur les œuvres poétiques qu'elle lit.

\section{Poètes russes et français du signe du temps}

Afin d'illustrer la présence de la « simplicité d'expression » dans la poésie contemporaine, l'auteur de Regain, née en Russie et ayant étudié en France, cite trois noms de poètes russes, Blok, Akhmatova, Essénine, et trois noms de poètes français, Jammes, Fort, Vildrac.

Alexandre Blok est né en 1880 à Saint Pétersbourg au sein d'un milieu d'intellectuels aisés. Son père est professeur de droit et musicien. Dans sa famille, on trouve aussi, du côté féminin, des écrivains et des traducteurs. À l'occasion de séjours dans le manoir familial, près de

\footnotetext{
${ }^{11}$ La plus grande partie dans le supplément du journal Davar, le reste dans des revues comme Ha-Shiloah ou Hèdim. À notre connaissance, ces articles n'ont jamais été traduits en français ni dans aucune autre langue.
} 
Moscou, il découvre la philosophie spiritualiste de son oncle, Vladimir Soloviev, et des poètes russes du XIX ${ }^{\mathrm{e}}$ siècle. En 1903, il épouse la fille du savant Mendeleïev. Après avoir commencé des études de droit, il s'oriente vers des études de lettres qu'il achève en 1906. Hanté par le destin de la Russie, le poète au caractère passionné, exigeant, qui était passé du symbolisme au fantastique, au rêve, au mysticisme, s'éteint en 1921, dans la ville de sa naissance, épuisé. Toujours sensible à la musique, sa poésie, où peuvent se mêler attirance pour la Révolution et images chrétiennes, trace la voie de la modernité poétique russe. Parmi ses œuvres majeures, on peut citer Cantiques de la Belle Dame (1904), Les Douze (1918) et parmi ses poèmes les plus célèbres, L'Inconnue et Les Scythes ${ }^{12}$.

Anna Akhmatova (de son vrai nom Gorenko) est née en 1888, huit ans après Blok, près d'Odessa, dans un milieu favorisé. Elle apprend le français et écrit très tôt de la poésie, influencée par Pouchkine. En 1910, elle épouse Nikolaï Goumilev, le fondateur du mouvement acméiste ${ }^{13}$, qui fera paraître ses premiers vers à Paris. Comme Blok dont elle deviendra l'amie, elle passe des études de droit aux études de littérature. Son premier recueil, Le Soir, paraît en 1912, suivi deux ans plus tard par Le Rosaire. En 1918, elle se sépare de son mari et épouse l'assyriologue Vladimir Chileïko. Trois ans plus tard, elle s'en sépare pour vivre, jusqu'en 1938, avec l'historien d'art Nikolaï Pounine. Étrangère à l'idéologie révolutionnaire régnante, sa parole poétique, considérée comme bourgeoise, fut interdite à partir des années 1920. C'est alors qu'elle traduit, pour vivre, Victor Hugo, Giacomo Leopardi, Rabindranath Tagore. La disparition de Staline permit sa lente réhabilitation. Deux ans avant sa mort, elle devint présidente de l'Union des écrivains. Elle s'éteint près de Moscou en 1966. Ses poèmes, tout empreints de lyrisme, sont souvent brefs, mais peuvent parfois se dérouler en vaste composition, comme Requiem. Ils nous parlent du temps qui passe, des souvenirs, du destin de la femme qui crée, de la vie éprise de liberté. Sa poésie, largement accueillie au sein de la société russe, allie le ton de la femme du peuple au raffinement de la culture, et touche le lecteur par sa sincérité, son intense émotion.

\footnotetext{
${ }^{12}$ Le premier évoque, comme un rêve, celle qui, près de la fenêtre assise, éveille le poète à des lointains merveilleux, de profonds mystères, des rivages bienheureux, et le second est une ode qui s'achève par un appel au vieux monde pour un avenir fraternel de travail et de paix. Blok qui avait mis tous ses espoirs dans la Révolution russe perdra ses illusions.

${ }^{13} \mathrm{~L}$ 'acméisme qui adopte une esthétique de la simplicité, influencera Rachel.
} 
Né en Russie centrale en 1895, au sein d'un milieu modeste, Serge Essénine, après avoir vécu sa petite enfance chez ses grands-parents, écrit ses premiers vers dès l'adolescence. En 1913, il travaille comme correcteur dans une imprimerie à Moscou et il suit des cours d'histoire, de littérature à l'université populaire. Il vit bientôt en ménage avec Anna Izriadnova qui lui donnera un enfant mais qu'il abandonnera rapidement. En 1915, à Saint-Pétersbourg, Alexandre Blok introduit Serge Essénine dans les milieux littéraires (il rencontre notamment Anna Akhmatova, Nikolaï Gounilev, Marian Tsvetaïeva). C'est en 1916 que parait son premier recueil, Radounitsa. Il s'enthousiasme pour la Révolution sans pouvoir oublier le passé. Il épouse Zinaïda Raïkh, secrétaire à La Cause $d u$ Peuple, avec laquelle il a deux enfants et qu'il quittera peu d'années plus tard. En 1918, paraît Golouben, son second recueil et, avec trois autres poètes, il fonde le mouvement imaginiste. Tout en continuant de donner des lectures, des récitals, il mène une existence dissipée, instable. Trois recueils, dont Transfiguration, voient le jour en 1920. En 1922, il épouse Isadora Duncan qu'il quitte un an plus tard. En 1923, il publie Poèmes d'un faiseur de scandales et il meurt à la fin de l'année $1925^{14}$. D'un tempérament sombre et nerveux, attiré par la boisson, ce poète a cherché à chanter avec lyrisme et réalisme la nature, l'amour, la terre natale, l'humanité, sur les traces d'un paradis perdu. De Serge Essénine, Rachel a traduit un poème en hébreu tandis qu'elle en a traduit quatre d'Anna Akhmatova ${ }^{15}$.

Le premier poète français que l'auteur de Regain cite à propos de la « simplicité d'expression » est Francis Jammes, auteur dont elle a traduit trois poèmes ${ }^{16}$ et auquel elle a consacré un article. Le poète pyrénéen est né à Tournay le 2 décembre 1868 et s'est éteint le jour de la Toussaint 1938 à Hasparren. Très jeune il avait perdu son père. Peu disposé pour les études ${ }^{17}$, il se tourne vers la poésie et est remarqué par Gide et Mallarmé. Le « Manifeste du Jammisme », publié au Mercure de France en 1897, le met sur l'avant-scène littéraire. En 1898, paraît son recueil le plus notoire, De l'Angélus de l'aube à l'Angélus du soir. Dans l'église de La Bastide-Clairence, en juillet 1905, tandis que Paul Claudel sert la messe, Francis Jammes se convertit au catholicisme, revient à la pratique

\footnotetext{
${ }^{14}$ C'était l'année où il s'était marié avec Sofia Soukhotina, la petite-fille de Léon Tolstoï.

${ }^{15}$ Cette dernière est l'auteur dont elle aura traduit le plus de poèmes.

${ }^{16}$ Prière pour aller au Paradis avec les ânes (Le Deuil des primevères) ; Il va neiger... et La salle à manger (De l'Angélus de l'aube à l'Angélus du soir).

${ }^{17}$ Lycéen à Pau, il échoue en 1888 au baccalauréat (avec un zéro en français).
} 
religieuse. C'est à l'automne 1907 qu'il épouse Geneviève Goedorp, lectrice fervente. De leur mariage naîtront sept enfants. Francis Jammes mène une existence bucolique tout en correspondant avec Mallarmé, Gide, Claudel... Jusqu'à sa mort, il écrit, publie, poésies, romans, compose une œuvre, conjuguant modernité et lisibilité, sous le sceau de l'humilité. Ce poète par excellence de la simplicité, qui est acquise et n'est plus un but chez lui, a été admiré hors de France par Kafka et Rilke. Parmi ses œuvres poétiques, on peut citer Le Deuil des primevères (1901) et après sa conversion, Clairières dans le ciel (1906), Les Livres des Quatrains (1923-1925).

Second poète français cité par Rachel au sujet de la simplicité, Paul Fort est né en 1872 à Reims. Son père, qui était agent d'assurances, emmène en 1878 la famille à Paris où Paul fréquente le lycée Louis le Grand. Alors que le XIX ${ }^{\mathrm{e}}$ siècle va s'achever, il décide de se vouer à la poésie et publie ses premiers vers. Avec Moréas et Salmon, il crée en 1905 la revue Vers et prose qu'il dirige avec Paul Valéry et où seront publiés Apollinaire, Max Jacob. Issu du symbolisme, élu «prince des poètes » en 1912, il écrit des Ballades françaises ${ }^{18}$ qui puisent leur inspiration dans la nature, l'histoire, l'existence quotidienne des hommes, usent d'une langue simple, parfois populaire et chantent, avec humour, fantaisie et mélancolie, le bonheur de vivre.

Dernier poète français cité par Rachel, Charles Vildrac (de son vrai nom Messager) est né à Paris, dans le Quartier Latin, en 1882. Son père avait participé à la Commune et avait été déporté en Nouvelle Calédonie. $\mathrm{Au}$ début du $\mathrm{XX}^{\mathrm{e}}$ siècle, alors que depuis l'adolescence il se passionne pour la poésie, il rencontre Georges Duhamel dont il épouse la sœur aînée, Rose, en 1905. C'est en 1906 que prend forme à Créteil, au lieu-dit Le Bras du Chapitre, le grand projet de l'Abbaye qui lui tenait à cour et où se trouvent fraternellement réunis lui-même, Georges Duhamel, les poètes René Arcos et Henri-Martin Barzum, le peintre Albert Gleizes. «Quelques jeunes hommes dont j'étais, exaltés de ferveur artistique et avides de remplir librement leurs jours selon leurs vœux, résolurent de bâtir leur Thélème, d'y assembler leurs vies et d'y subsister » ${ }^{19}$. Après

\footnotetext{
${ }^{18} 40$ volumes de 1897 à 1958.

${ }^{19}$ Charles Vildrac, Livre d'amour, Paris, Seghers, Le Temps des Cerises, 2005, L'Abbaye, Avertissement, p. 165. (Thélème fait référence à Rabelais cité en exergue). « Je rêve l'Abbaye hospitalière / À tous épris d'art » - « [...] je rêve l'Abbaye / Gaie et recueillie, / Où vivre libres, en thélémites passionnés » (Ibid., L'Abbaye II, p. 175). "Vivre en amour, vivre en ferveur / En la maison des chers labeurs, / En l'abbaye hospitalière » (Ibid., L'Abbaye III, p. 180). Cette communauté d'artisans, d'artistes, de
} 
l'expérience exaltante de vie communautaire de l'Abbaye, l'auteur du Livre d'amour $(1910)^{20}$ ouvre avec sa femme une galerie rue de Seine où sont exposés Friez, Marquet, Vlaminck. Dans les années vingt, il se fait connaître comme auteur de théâtre. Sous l'Occupation, alors qu'il participe à la publication clandestine des Lettres françaises, il est arrêté par la Gestapo et emprisonné à Fresnes. Après la mort de sa femme, il épouse en 1970 Suzanne Rochat, la traductrice en italien de ses œuvres. Ce poète, apôtre d'une pensée libre et humaniste, rêveur d'un avenir de sagesse et de paix, s'éteint à Saint Tropez au début de l'été 1971. Comme la poésie de Rachel, la poésie de Charles Vildrac est simple et directe, inséparable de la vie, centrée sur l'homme, attentive au mystère, et un lyrisme retenu y fait sourdre une douce émotion. Comme elle, il pense que la simplicité est voie de fraternité et rejette le factice, l'artifice, ce qu'il appelle les «littérateurs littératurant ${ }^{21}$. Soucieux de sincérité, d'authenticité, libre à l'égard des règles prosodiques, en quête d'une Terre promise, d'un Eden perdu, il chante la nature, l'enfance, les hommes pauvres, l'amour, l'amitié, la mort et la vie ${ }^{22}$.

\section{Poètes hébreux et signe du temps}

À l'opposé des trois poètes russes et des trois poètes français dont elle évoque le nom dans le paragraphe précédent, le poète Bass "échappe [...] au signe du temps" aux yeux de Rachel. Fils d'un professeur d'hébreu, Samuel Bass est né en Ukraine en 1899. Sa famille émigre en Palestine en 1906. Il étudie dans les écoles de Jaffa et de Tibériade - c'est alors que son talent littéraire se révèle - avant d'enseigner à Tibériade puis à Tel-Aviv où il est directeur d'école. En 1923, il épouse Manna Shapiro, fille de Zacharie Shapiro ${ }^{23}$. Son fils unique, Adam Bass, empruntera la voie de son père en devenant directeur d'école et en publiant des histoires pour enfants. Au printemps 1949, Samuel Bass est victime d'une hémorragie cérébrale au cours d'une

\footnotetext{
pèlerins des mots, qui durera un peu plus d'un an, trouvait son unité dans le service du Beau. La communauté du kibboutz, à laquelle Rachel a appartenu dans les années 1920, s'unifiait autour du travail de la Terre. La communauté des moines, modèle de la communauté laïque de Vildrac et de Duhamel, s'harmonisait dans le service du Ciel.

${ }^{20}$ La dernière édition revue par l'auteur paraîtra en 1959. En 1907 était paru son recueil Images et Mirages. En 1968 sera publié Pages de journal.

${ }^{21}$ Livre d'amour, Je rêve l'Abbaye, p. 186.

${ }^{22} \mathrm{Au}$ danger de « prosaïsme » que pointe Rachel dans son article et qui « menace » « le chemin de la simplicité », il arrivera parfois à ces trois poètes français de ne pas échapper.

${ }^{23}$ Après la mort de son épouse, il se marie avec Sephora Danim.
} 
leçon et décède à l'hôpital de Tel-Aviv. Dès son jeune âge, il a écrit et publié. Attentif à l'éducation, il crée une revue pour la jeunesse. Son œuvre se compose de poèmes ${ }^{24}$, de contes pour enfants et d'essais de critique littéraire. Parmi ses recueils, on peut citer Adam (1927) auquel se réfère Rachel, Rivages (1933), Fleuve antique, Poèmes bibliques $(1941)^{25}$. Sa poésie évoque la nature et l'enfance, les saisons et les fêtes. Rachel lui reproche son déracinement à l'égard de son lieu, de son temps, tout en lui reconnaissant une aptitude à dessiner avec assurance des paysages.

Autre exemple de poète étranger au signe du temps choisi par Rachel, Mordekhaï Temkin. Ce dernier, né en Pologne en 1891, est monté au Pays en 1907, avec son frère Moïse, dans le cadre de la seconde aliyah. Comme Rachel, il commence par écrire de la poésie qui dépeint les paysages découverts alors, la vie des pionniers. Il fait partie des écrivains du mensuel littéraire Hèdim qui voit le jour dans les années vingt. Temkin nourrit aussi sa poésie des pogroms et des événements de la Révolution. Ses traductions d'œuvres classiques écrites en allemand, comme Les Buddenbrook de Thomas Mann, Peter Schlémihl d'Adalbert von Chamisso et Henri le Vert de Gottfried Keller, contribuent à le faire connaître. Dans les années quarante, il travaille comme professeur à Jérusalem. Il meurt en 1960. En 1927 était paru son recueil Gouttes qu'évoque Rachel et en 1934, Poèmes et prières. Récemment, en 2013, a été publiée une anthologie de sa poésie, avec des inédits, sous le titre de Fleurs blanches de solitude. Rachel reproche à Temkin son indifférence au signe du temps, dont la simplicité d'expression est la marque essentielle, mais souligne la qualité picturale et musicale de sa poésie.

L'auteur de Regain se tourne alors vers Shlonsky pour y chercher, en le lisant, le relisant, le signe du temps comme une Toison d'or. D'une famille juive hassidique cultivée et artiste, Abraham Shlonsky est né en Ukraine en 1900 et s'est éteint à Tel-Aviv en 1973. En 1921, il s'est installé définitivement en Palestine où il participe à la fondation du kibboutz Ein-Harod. Militant au sein d'un mouvement d'inspiration marxiste, il s'oppose à la poésie traditionnelle et défend une poésie moderne, renouvelée, à distance de la poésie de Bialik et de sa génération. Par ses traductions de Shakespeare, Gogol, Tchekhov,

\footnotetext{
${ }^{24}$ Parmi les plus significatifs, Mon village, Bientôt descendit sur nous le beau jour $d u$ sabbat, L'été est passé, Bergeronnette.

${ }^{25}$ Une vingtaine d'années après sa mort est publié Jusqu'à la fin du chemin, Chants et ballades (1970).
} 
Pouchkine..., il participe à la renaissance de la langue hébraïque. Dans son poème Détresse, il déplore le destin des Juifs victimes de pogroms en Ukraine au cours de la Révolution. Parmi ses écrits, outre Père-Mère (1927), cité par Rachel, figurent Tu ne tueras pas (1930), Poèmes (1954), En face du désert (1973). En 1967, il reçoit le Prix Israël de la littérature. Rachel lui pardonne ses défauts, ses erreurs, car il a su habilement conjuguer « simplicité d'expression et déluge d'images ».

Dernier poète à être évoqué dans l'article Sur le signe du temps: Élisheva. Élisheva Bichovsky (nom de plume d'Élizaveta Zhirkova) est née à Riazan (Russie) en 1888 d'un père membre de l'église provoslave et d'une mère catholique irlandaise. Initiée à la culture et aux traditions juives par de jeunes juives, elle étudie le yiddish puis l'hébreu. Elle traduisit du yiddish au russe des poèmes de Juda Halévi, traduits auparavant de l'hébreu au yiddish par Bialik; de l'hébreu au russe Gershon Shofman et Yossef Haïm Brenner. Après avoir d'abord écrit des poèmes en russe, elle publie ses premiers poèmes en hébreu dès 1920, année où elle épouse Simon Bichovsky, professeur, critique littéraire, éditeur. Tous deux partent pour la Palestine en 1925 avec leur fille Miriam. Non-juive ayant quitté son pays et sa langue pour rejoindre le peuple juif au Pays, elle y fut bien accueillie pour son courage, son humilité et ses qualités poétiques. Elle se rapproche du groupe littéraire dirigé par Bialik et s'éloigne du groupe des nouveaux poètes dirigé par Shlonsky. Elle publie alors ses œuvres (poésie, critique littéraire, essais) en hébreu. Influencée par l'acméisme, elle n'en poursuit pas moins une voie propre et sa poésie, qui oscille entre sentiment d'exil et recherche de la terre promise, aime prendre le ton de la confession. En 1932, elle perd son mari. Confrontée à une situation financière difficile, elle vit de petits emplois alimentaires ${ }^{26}$, dans la pauvreté, à l'écart désormais des milieux littéraires et ne publiera plus jusqu'à sa mort en 1949. Bien que non convertie au judaïsme, elle repose au cimetière juif de Kinnéret (Tibériade), près de la tombe de Rachel... Parmi les œuvres de celle qui était appelée la « Ruth des rives de la Volga », on peut citer À la lumière du matin, un chant d'allégresse (1926), Histoires (1928), Poète et homme, essai sur la poésie d'Alexandre Blok (1929). Dans la poésie d'Élisheva Bichovsky, Rachel goûte la «simplicité d'expression » tout en excusant son absence parfois de force ${ }^{27}$.

\footnotetext{
${ }^{26}$ Bialik lui apporte une aide.

${ }^{27}$ Dans une des lettres que Rachel a écrites à Shulamite Klugai et publiées en avril 2013 par les Archives nationales d'Israël à l'occasion du $82^{\mathrm{e}}$ anniversaire de sa mort, elle
} 


\section{L'ordre de la simplicité et de la puissance}

L'idéal esthétique de Rachel, celui qu'elle admire quand elle le sent incarné chez les poètes qu'elle lit, celui qu'elle s'attache elle-même à mettre en pratique avec constance dans sa propre poésie, c'est l'alliance de la simplicité et de la force. La beauté du poème jaillit quand le simple se mêle au puissant. Telle est la source de la véritable poésie, la poésie de l'émotion poignante, du lyrisme ardent, celle qui revient sans cesse à l'homme, au cœur de l'homme. Il serait bien superficiel de penser que le simple se confond avec le facile. "À la vérité il est difficile le chemin de la simplicité ». La juste simplicité navigue entre deux écueils opposés : le prosaïsme et l'affèterie. Si le poète en recherche d'un langage simple bascule dans le trivial, le banal ou dans l'élégance, les ornements, il s'éloigne en réalité de la simplicité lyrique authentique. Ni indigence, ni excès du signifiant, mais des mots, une syntaxe, sobres, dépouillés, qui laissent éclater la lumière du signifié. Le style où s'allient avec bonheur simplicité et puissance n'est pas un style conformiste, académique, mais un style qui transforme, bouleverse, un style qui, en nous éloignant de toutes nos habitudes, nous donne à voir autrement le monde. Un style qui nous éloigne des « littérateurs littératurant » dénoncés par Vildrac.

En conclusion de son article, Rachel enracine la vertu esthétique du chemin de la simplicité dans la Bible en modifiant quelques mots d'un verset essentiel du célèbre Songe de Jacob: «En vérité, il y a une nouvelle magnificence d'expression en ce lieu et je ne le savais pas $! »^{28}$. La Présence du Seigneur devient la beauté de l'expression. La Shekhinah se dévoile dans la tiph'érét bitouy (magnificence d'expression). Au théologique ou au mystique Rachel donne une résonance esthétique, poétique.

\section{Sur le signe du temps (présentation et traduction de l'hébreu)}

L'article Sur le signe du temps donne comme la clé de l'esthétique de Rachel : l'alliance de la simplicité et de la puissance. Et s'il fallait hiérarchiser ces deux vertus stylistiques, il faudrait mettre la simplicité

évoque une soirée littéraire qu'elle a organisée à Tel-Aviv chez son frère Jacob Blaustein-Séla et à laquelle étaient présentes les écrivains-poètes Yocheved BatMyriam, Anda Pinkerfeld et Élisheva... C'est un signe de la proximité qui existait entre cette dernière et l'auteur de Regain.

${ }^{28}$ Voir Genèse 28,16 . Rachel reprend les mots de ce verset en remplaçant « il y a le Seigneur [yèsh Adonai]» par «il y a une nouvelle magnificence [ou beauté] d'expression ». 
au-dessus de la force, comme le laisse entendre ce qu'écrit Rachel à la fin de son article à propos d'Élisheva. Mais tant qu'un poème ne sera pas, à la fois, écrit et de manière simple et de manière forte, il ne pourra atteindre la vraie beauté, la « magnificence d'expression ».

Cet article offre aussi l'intérêt de montrer combien Rachel était une grande lectrice de poésie hébraïque, russe et française, de dresser un panorama vivant des œuvres poétiques publiées en Palestine et ailleurs, à son époque. Comme lectrice, elle sait à la fois témoigner d'enthousiasme et d'une réelle capacité de recul pour dégager des critères de jugement esthétique. Si Rachel est incontestablement poète, on ne doit pas ignorer qu'elle est aussi une critique littéraire exigeante, originale et convaincante. Sa riche personnalité de poète se double d'une vive flamme critique. C'est un aspect de son œuvre qu'il convient ainsi de mettre en lumière.

\section{Rachel : Sur le signe du temps}

Voici qui est clair pour moi : le signe du temps dans l'art poétique est la simplicité de l'expression. Expression simple, c'est-à-dire : expression des premiers tremblements de l'émotion lyrique, expression immédiate, avant qu'il soit possible de couvrir sa nudité dans des vêtements de luxe, soie et parures d'or ; expression dépouillée d'artifices littéraires, qui touche le cœur par sa vérité humaine, qui apaise par sa fraîcheur, dont la force permet de se graver dans la mémoire, de nous accompagner dans la vie de chaque jour et de chanter soudain de joie.

Ne trouve-t-on pas cette simplicité d'expression, ou du moins un effort pour tendre vers elle, manifeste dans nombre d'œuvres poétiques contemporaines, chez les Russes (Blok, Akhmatova, Essénine), chez les Français (Jammes, Paul Fort, Charles Vildrac) ? Jusqu'à se révolter contre la rime et la strophe sacrées, jusqu'à se défier du rythme musical ordinaire?

Quand je feuillette un recueil poétique de Bass («Adam », éditions Hèdim), je pense : «Mon Dieu ! Comment a-t-il réussi à échapper au signe du temps?». Le détachement du sol propre au temps est à condamner tout comme le détachement du sol propre au lieu. Les étendues sans fin ne nous conviennent pas, nous cherchons un coin où vivre clôturé. 
On trouve chez Bass des sonnets sans relief, des contours de paysages dessinés d'une main assurée, une riche palette de couleurs qui lui est propre, seulement « la couleur du temps » lui a manqué.

En ce qui concerne Temkin («Gouttes », éditions Ketouvim), voici sa voie : une image s'élève des pages de son livre, une image d'homme à côté du chemin, de chagrin crépusculaire, de pitié fraternelle. Pas un son faux n'écorche les oreilles, toutefois lui aussi, quoique moins que Bass, il se montre indifférent au signe et tu prends «Père - Mère » (A. Shlonsky, «Père - Mère », éditions Ketouvim) et tu lis et relis, tu es prêt à pardonner à Shlonsky ses erreurs, en raison de son habileté à être tellement fils de son époque.

Il me semble qu'il n'y a pas ici de difficulté : simplicité d'expression et déluge d'images, de métaphores. - Comment cela ? Du fait que ces paroles peuvent relever non seulement de la magie de la langue des hiéroglyphiens ${ }^{29}$ de notre pays mais aussi représenter des métaphores qui sont la conséquence directe d'une vision du monde poétique, comme ceci : œil disposé ainsi et non autrement, émotion s'élançant pitoyable avec son vêtement, exemple d'enfants « qui ont de la chance ", nés avec une tunique.

Une simplicité d'expression qui n'est pas toujours mêlée avec une puissance d'expression, mais qui fait toujours absoudre son manque de puissance (par exemple dans les poèmes d'Élisheva). En revanche si la puissance en vient à fléchir par manque de simplicité, ceci ne sera pas excusé. À la vérité il est difficile le chemin de la simplicité. D'une part le menace le prosaïsme et d'autre part - l'affèterie. Elle transforme toutes nos conceptions habituelles. La conscience de son mérite nous est venue directement, à travers une lecture inattendue sur les traces de notre père, Jacob : «En vérité, il y a une nouvelle magnificence d'expression en ce lieu et je ne le savais pas ! » ${ }^{30}$.

\footnotetext{
${ }^{29}$ Ceux qui écrivent de manière hiéroglyphique (hartoumim), comme des mages, des devins.

${ }^{30}$ Songe de Jacob (Gn 28,16).

31 Je remercie Isabelle Dotan (Université Bar-Ilan, Israël) et Liliane Apotheker (International Council of Christians and Jews) pour leur aide dans la résolution des difficultés du texte hébreu.
} 
Tombe de Rachel près du lac Kinnéret

(Photo d'Isabelle Dotan)

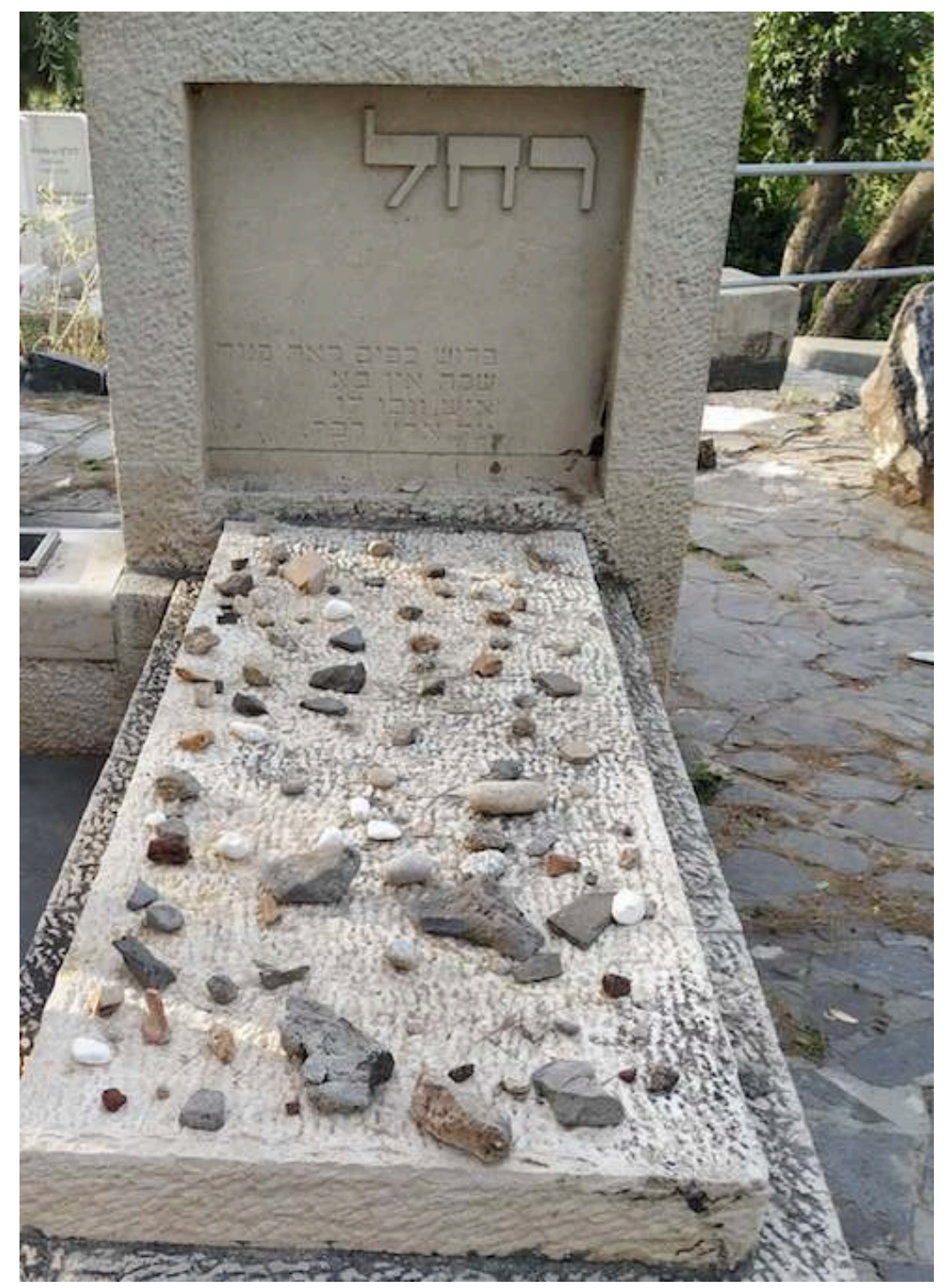

